\title{
M2-Type Macrophages Induce Tregs Generation by Activating the TGF- $\beta /$ Smad Signalling Pathway to Promote Colorectal Cancer Development
}

\author{
Xueqian $\mathrm{Ma}$ (iD \\ Yu Gao ${ }^{2}$ \\ Yanlei Chen' \\ Jinhao Liu' \\ Chunyu Yang ${ }^{3}$ \\ Cuifen Bao ${ }^{4}$ \\ Yanping Wang' \\ Yang Feng (D) \\ Xiaoyu Song' \\ Shifeng Qiao (D) \\ 'The Second Ward of Colorectal Surgery, \\ The First Affiliated Hospital of Jinzhou \\ Medical University, Jinzhou, 121000 , \\ Liaoning, People's Republic of China; \\ ${ }^{2}$ Computer Teaching and Research \\ Section, Jinzhou Medical University, \\ Jinzhou, I21000, Liaoning, People's \\ Republic of China; ${ }^{3}$ Department of \\ Pathology, The First Affiliated Hospital of \\ Jinzhou Medical University, Jinzhou, \\ 121000, Liaoning, People's Republic of \\ China; ${ }^{4}$ Basic Medical Experimental \\ Teaching Center, Jinzhou Medical \\ University, Jinzhou, 121000 , Liaoning, \\ People's Republic of China
}

Correspondence: Shifeng Qiao

The Second Ward of Colorectal Surgery, The First Affiliated Hospital of Jinzhou Medical University, No. 2 Section 5,

Renmin Street, Guta District, Jinzhou,

Liaoning, 12100I, People's Republic of

China

Tel +86 15904161717

Email shifengqiao2020@163.com
Purpose: The increase of both M2-type macrophages and Tregs is closely associated with the development of colorectal cancer. However, the mechanism of their interaction is still unclear. In this study, we investigated the correlation of M2-type macrophages with Tregs and the possible mechanisms between them.

Methods: Using immunohistochemistry, we analysed Smad3 (a key protein in the TGF- $\beta$ / Smad signalling pathway) expression in colorectal cells, as well as infiltrating numbers of CD163 (a marker for M2-type macrophages), Foxp3 (a marker for Tregs) in 250 surgically resected colorectal cancer tissues, matched normal and paracancerous tissues. The relation of CD163 and Foxp3 was investigated in CRC with clinicopathological characteristics and preoperative tumour markers.

Results: CD163, Foxp3 and Smad3 were upregulated in CRC tissues compared to matched normal and paracancerous tissues. Interestingly, CD163 and Foxp3 were significantly positively correlated in CRC, and both were significantly positively correlated with Smad3. Both CD163 and Foxp3 were upregulated with increasing tumour TNM staging, increasing number of lymph node metastases and increasing vascular invasion. Additionally, CD163 was upregulated with increasing depth of infiltration. The number of M2-type macrophages and the expression levels of preoperative CEA, CA19-9 and CA72-4 were significantly positively correlated. The number of Tregs was significantly positively correlated with the expression levels of preoperative CEA and CA19-9.

Conclusion: M2-type macrophages may induce Tregs generation through activation of the TGF- $\beta$ /Smad signalling pathway, which can promote the development of colorectal cancer. Keywords: M2-type macrophages, Tregs, TGF- $\beta$ /Smad signalling pathway, CD163, Foxp3, colorectal cancer, CRC

\section{Introduction}

Colorectal cancer (CRC), currently one of the most morbid and mortal malignant tumours worldwide, is increasing in incidence and mortality year by year. By 2035 , the total number of deaths from rectal and colon cancer will be expected to increase by $60 \%$ and $71.5 \%$, respectively. ${ }^{1}$ Recent studies ${ }^{2-4}$ have shown that specific immune cells in the tumour microenvironment (TME) play a key role in disease progression, significantly influencing tumour development, therapeutic effect and clinical outcomes.

M2-type macrophages and regulatory T cells (Tregs) are crucial players in the TME. They can promote tumour growth and immune tolerance. ${ }^{5}$ The presence of 
M2-type macrophages has been associated with poor clinical outcomes in almost all malignant tumours and is thought to affect disease outcomes by stimulating angiogenesis, lymphangiogenesis, immune suppression and possibly by decreasing the effectiveness of certain treatments. ${ }^{6,7}$ Cluster of differentiation 163 (CD163) is a specific marker for M2-type macrophages. ${ }^{8}$ Tregs, a kind of CD4+ T lymphocyte, may suppress anti-tumour functions and regulate immune tolerance. ${ }^{9}$ Alterations in their development, function or homeostasis may predispose them to a variety of diseases including autoimmunity, graft rejection and cancer. ${ }^{10}$ Forkhead transcription factor p 3 (Foxp3), which plays a key role in the differentiation and function of Tregs, is a specific marker for Tregs. ${ }^{11}$ The correlation between M2-type macrophages and Tregs has been demonstrated in ovarian, laryngeal, prostate and nasopharyngeal cancers. ${ }^{6,12-14}$ However, it has not been reported in CRC.

Epithelial-mesenchymal-transition (EMT) is an important process in tumour metastases. During the time the epithelial cells lose their adhesive properties and acquire a fibroblast-like morphology, which can gain the ability to invade, migrate and resist treatment. ${ }^{15}$ EMT is a multicascade complex process. Signalling pathway is strongly associated with EMT, especially the transforming growth factor- $\beta$ (TGF- $\beta$ )/drosophila mothers against decapentaplegic protein (Smad) signalling pathway. ${ }^{16,17}$ It has been reported that M2-type macrophages can activate the TGF- $\beta /$ Smad signalling pathway by secreting TGF- $\beta$, which promotes EMT in tumour cells. ${ }^{18,19}$ Additionally, studies found that the combination of Smad3 and Foxp3 enhancer site plays a decisive role in inducing the differentiation of naive CD4+ T cells to Tregs. ${ }^{20,21}$

In this study, we analysed the correlation among the expression of M2-type macrophages, Tregs and Smad3, a key protein of the TGF- $\beta /$ Smad signalling pathway, in colorectal tissues of 250 CRC patients through IHC. We hypothesised that M2-type macrophages may activate the TGF- $\beta /$ Smad signalling pathway by secreting TGF- $\beta$, leading to increased expression of Foxp $3+$ Tregs. This study aimed to understand the possible related mechanisms of CRC infiltration and metastases and to explore potential new therapeutic targets for new immunotherapy in CRC, which lays a foundation for further studying the immune pathogenesis of malignant tumours and exploring new immunotherapy targets. It has particularly important clinical value for early diagnosis, treatment and prognostic evaluation of CRC.

\section{Materials and Methods}

\section{Patients and Data Collection}

A total of 368 patients with CRC admitted to The Second Ward of Colorectal Surgery, The First Affiliated Hospital of Jinzhou Medical University from January 2020 to December 2020 were selected for this study. According to the inclusion and exclusion criteria, 250 patients of them were included. The clinical data of these patients are shown in Table 1.

The postoperative pathological tissue specimens of 250 patients with CRC were divided into three groups: CRC tissues, normal and paracancerous colorectal tissues. The above three groups of tissue specimens were obtained from the non-necrotic area in the center of the cancer foci, the area $10 \mathrm{~cm}$ from the cancer margin and the area $5 \mathrm{~cm}$ from the cancer margin of the same patient, respectively.

Inclusion criteria: (1) primary CRC; (2) diagnosis and first surgical treatment in our hospital; (3) consent to participate in this study. Exclusion criteria: (1) received chemotherapy, radiotherapy or immune-targeted therapy after diagnosis; (2) had multiple sites of intestinal malignant tumours or combined with other systemic malignant tumours.

\section{Experimental Methods}

The expression profiles of CD163, Foxp3 and Smad3 in different colorectal tissues of the same patient were detected by immunohistochemical (IHC). The experimental steps

Table I Clinical Characteristics of the 250 CRC Patients

\begin{tabular}{|l|c|c|}
\hline Variables & $\mathbf{n = 2 5 0}$ & Percent (\%) \\
\hline Age (years) & 105 & 42.00 \\
$<65$ & 145 & 58.00 \\
$\geq 65$ & & \\
\hline Gender & 153 & 61.20 \\
Male & 97 & 38.80 \\
Female & & \\
\hline TNM staging & 37 & 14.80 \\
I & 108 & 43.20 \\
II & 77 & 30.80 \\
III & 28 & 11.20 \\
IV & & \\
\hline Histologic types & 232 & 92.80 \\
Tubular adenocarcinoma & 12 & 4.80 \\
Mucinous carcinoma & 6 & 2.40 \\
Other types & & \\
\hline
\end{tabular}


were as follows. All specimens were formalin-fixed, paraffin-embedded and cut into $4 \mu \mathrm{m}$ thick sections. First, after dewaxing with xylene and hydration with ethanol, antigen retrieval was conducted using EDTA Antigen Retrieval Solution PH=8.0 (ZLI-9072, ZSGB-Bio, Beijing, China) in a pressure cooker for 2.5 minutes. Subsequently, Endogenous Peroxidase-blocking Solution (KIT-9710, Maixin-Bio, Fuzhou, China) was added and incubated for 10 minutes at room temperature, followed by Non-specific Stainingblocking Solution (KIT-9710, Maixin-Bio, Fuzhou, China) for 30 minutes at room temperature. Third, Anti-CD163 monoclonal antibody (1:500, ab182422, Abcam, Cambridge, UK), Anti-Foxp3 monoclonal antibody (1:500, ab20034, Abcam, Cambridge, UK) and Anti-Smad3 monoclonal antibody (1:500, ab40855, Abcam, Cambridge, UK) were added and incubated in a refrigerator at $4{ }^{\circ} \mathrm{C}$ for 16 hours. Fourth, Biotin-Labeled Goat Anti-Rabbit/Mouse IgG polymer (KIT-9710, Maixin-Bio, Fuzhou, China) and Streptavidin Peroxidase (KIT-9710, Maixin-Bio, Fuzhou, China) were added sequentially and incubated in a $37{ }^{\circ} \mathrm{C}$ water bath for 30 minutes. Fifth, the pathological sections were stained with a DAB kit (DAB-0031, Maixin-Bio, Fuzhou, China) and Hematoxylin (G1140, Solarbio, Beijing, China). Finally, they were dehydrated and sealed. PBS (ZLI-P063, ZSGB-Bio, Beijing, China) was used for washing in all steps.

\section{Judgment of Staining Results}

CD163+ staining was expressed as the presence of tan or yellow particles in the cell membrane or cytoplasm of M2type macrophages. Foxp3+ staining was expressed as the presence of tan or yellow particles in the nucleus of Tregs. Smad3 + staining was expressed as the presence of tan or yellow particles in the cell membrane or cytoplasm of colorectal cells. Known positive results were used as a positive control. PBS was used as a negative control instead of primary antibody.

Five high magnification fields (400x) were randomly selected from the stained pathological sections. Image $\mathrm{J}$ was applied to determine the positive cells, which were then confirmed by two pathologists under double-blind methodological conditions.

For pathology sections stained with Anti-CD163 monoclonal antibody and Anti-Foxp3 monoclonal antibody, the mean number of positive cells was calculated for the five high magnification fields, and this mean was the final score for this section. For pathology sections stained with AntiSmad3 monoclonal antibody, the average of the staining indices of the five sections was calculated, and this average was the final score for this section. (Staining Indices $=$ the staining intensity $\times$ the percentage of positive cells). The staining intensity was divided into four levels: 0 (negative), 1 (weak), 2 (moderate), 3 (strong). The percentage of positive cells was regarded as $0(0-10 \%), 1$ (11-25\%), 2 (2640\%), 3 (41-75\%), 4 (76-100\%).

Taking the median of the final score as the boundary, the final score greater than or equal to this median is a high expression, and the final score less than this median is low expression.

\section{Statistical Analysis}

Statistical analysis was performed using SPSS 26.0. The Kruskal-Wallis $H$-test was used to compare means among multiple groups. The independent sample $t$-test was used to compare means between the two groups. The $\chi^{2}$ test was used to compare count data. Spearman correlation analysis and linear regression analysis were used to analyze the correlation between two variables. A value of $p<0.05$ was considered significant.

\section{Results}

\section{The Expression of CD 163, Foxp3 and Smad3 in Normal, Paracancerous and CRC Tissues}

IHC results showed that CD163 mainly infiltrated in the tumour mesenchyme in cancer tissues, and it expressed the membrane and cytoplasm of M2-type macrophages, which was brownish-yellow (Figure 1A). CD163 mainly infiltrated the mucosal mesenchyme in normal and paracancerous tissues, which was light yellow (Figure 1B and C). Foxp3 was more distributed in the tumour mesenchyme in cancer tissues, and it expressed the nucleus of Tregs, which was brownish-yellow (Figure 1D). In normal tissue and paracancerous tissues, Foxp3 had a small extent in the mucosal interstitium, which was light yellow (Figure 1E and F). Smad3 was mainly expressed on the cell membrane and cytoplasm of colorectal cells, which stained differently in normal, paracancerous and CRC tissues (Figure 1G-I).

\section{The Difference Between CDI63, Foxp3 and Smad3 in Normal, Paracancerous and CRC Tissues}

The Kruskal-Wallis $H$-test was used to compare the expression differences of CD163 $(\mathrm{H}=220.487, p=0.000)$, Foxp3 


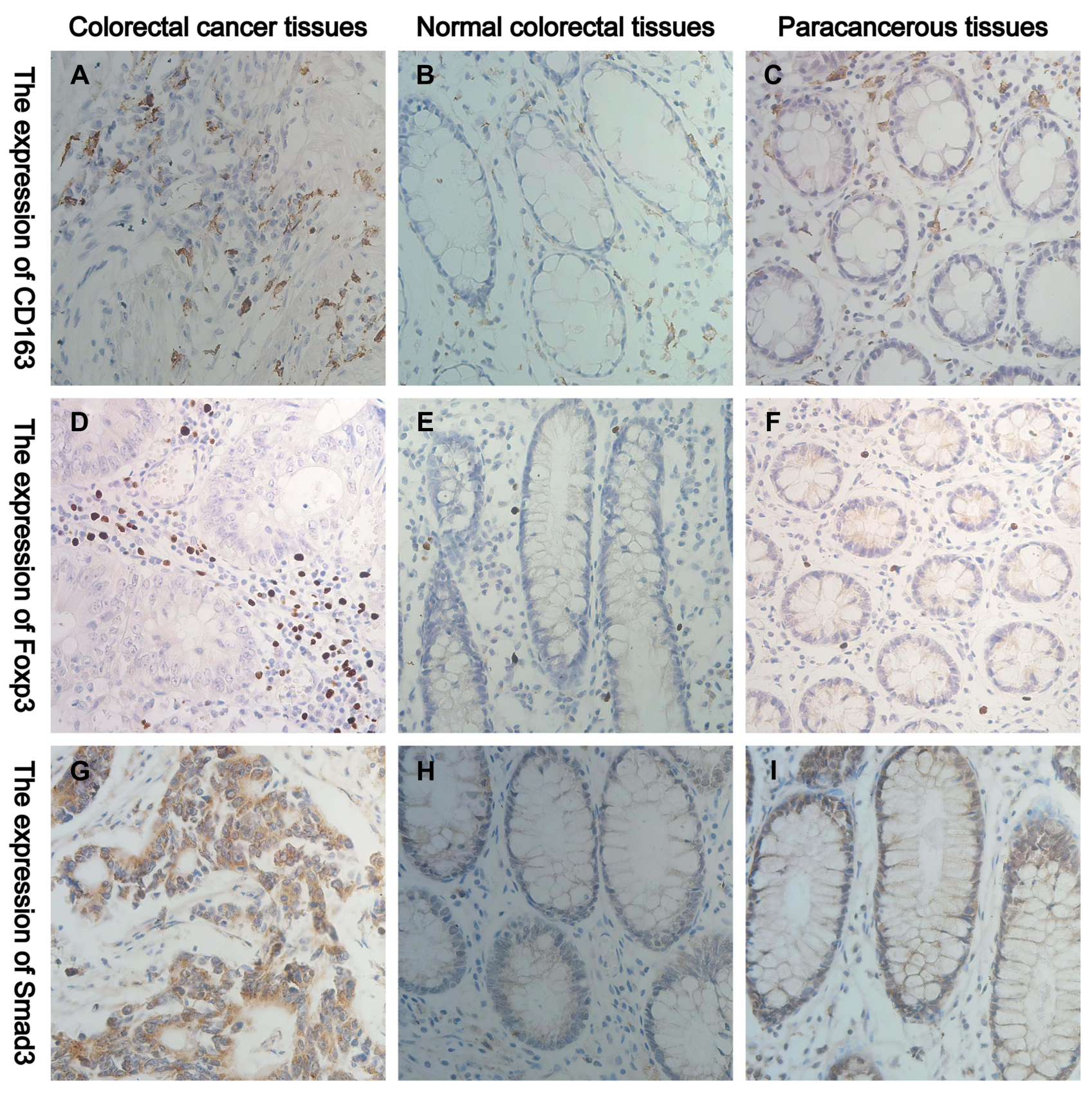

Figure I Representative IHC staining of CDI63, Foxp3 and Smad3 in normal, paracancerous and CRC tissues (400× field). (A-C) The cell membrane and cytoplasm of M2type macrophages are stained brown. (D-F) The nucleus of Tregs are stained brown. (G-I) The cell membrane and cytoplasm of Smad3+ cells are stained brown.

$(\mathrm{H}=198.303, p=0.000)$ and $\operatorname{Smad} 3(\mathrm{H}=130.486, p=0.000)$ in the three groups of colorectal tissues. The independent samples $t$-test was applied to analyse the numbers of CD163, Foxp3 and Smad3 in colorectal tissues among the three groups. The expression of CD163 $(\mathrm{t}=-16.331, p=0.000$; $\mathrm{t}=$ $-15.672, p=0.000)$, Foxp3 ( $\mathrm{t}=-15.616, p=0.000 ; \mathrm{t}=-14.261$, $p=0.000)$ and Smad3 ( $\mathrm{t}=-11.513, p=0.000 ; \mathrm{t}=-10.122$, $p=0.000$ ) was increased in CRC tissues compared with normal and paracancerous tissues. However, the expression of the above three kinds of proteins between normal and paracancerous tissues had no statistically significant difference $(\mathrm{t}=-1.959, p=0.051 ; \mathrm{t}=-1.842, p=0.066 ; \mathrm{t}=-1.957, p=0.051)$

(Figure 2).

\section{The Relation of CDI63 and Foxp3 with} Clinicopathological Characteristics in CRC To further explore the possible role of M2-type macrophages and Tregs in CRC, we used the $\chi^{2}$ test to compare 

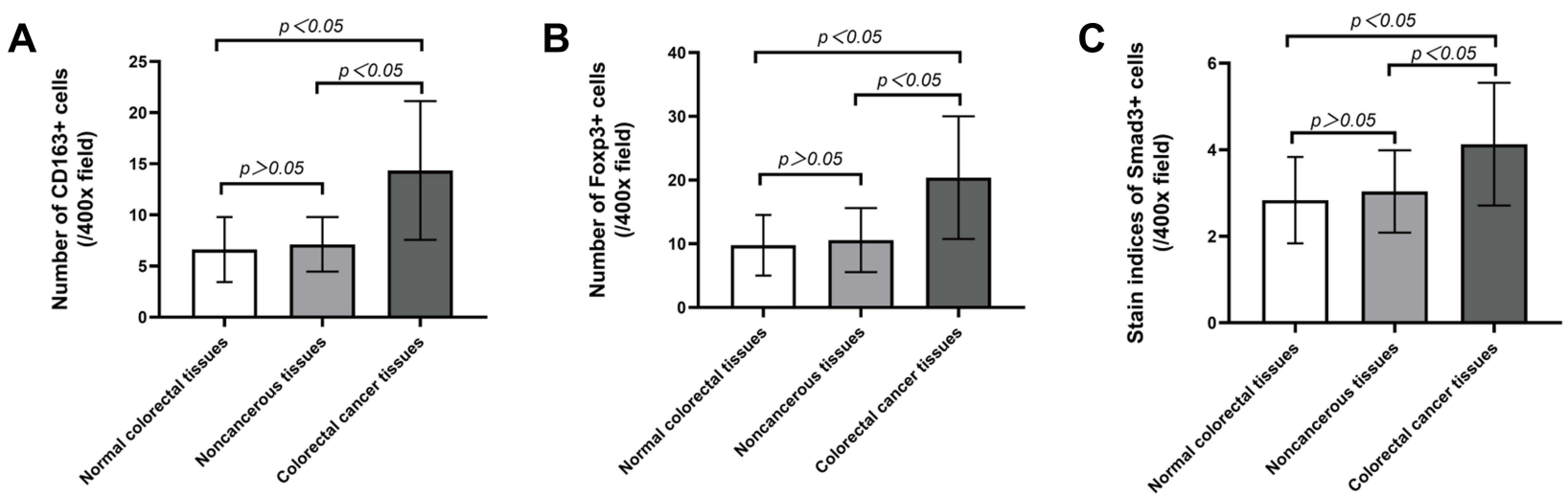

Figure 2 The expression levels of CDI63, Foxp3 and Smad3 are different in normal, paracancerous and cancer tissues. (A) The number of CDI63 in CRC tissues, their matched normal and paracancerous tissues. (B) The number of Foxp3 in CRC tissues, their matched normal and paracancerous tissues. (C) The staining indices of Smad3 in CRC tissues, their matched normal and paracancerous tissues.

CD163 and Foxp3 with the clinicopathological characteristics. The results showed that CD163 in CRC tissues was related to the patient's tumour TNM staging $\left(\chi^{2}=19.397\right.$, $p=0.000)$, number of lymph node metastases $\left(\chi^{2}=9.862\right.$, $p=0.019)$, depth of infiltration $\left(\chi^{2}=9.881, p=0.019\right)$ and vascular invasion $\left(\chi^{2}=4.993, p=0.026\right)$. The expression level of Foxp3 in CRC tissues was related to the patient's tumour TNM staging $\left(\chi^{2}=9.056, p=0.029\right)$, number of lymph node metastases $\left(\chi^{2}=9.177, p=0.027\right)$ and vascular invasion $\left(\chi^{2}=4.278, p=0.039\right)$ (Table 2).

\section{The Correlation of CDI63, Foxp3 and Smad3 with Different Tumour TNM Staging in CRC}

By analysing the expression of CD163 and Foxp3 in CRC tissues, we found that they are most closely related to tumour TNM staging. Comparing the expression levels of CD163, Foxp3 and Smad3 in CRC stages I, II, III and $\mathrm{IV}$, we found that the above three proteins were different in CRC patients with different TNM staging (Figure 3A-L). With the increase of tumour TNM staging, the expression of CD163, Foxp3 and Smad3 in CRC also gradually increased $\left(\chi^{2}=19.397, \quad p=0.000 ; \quad \chi^{2}=9.056, \quad p=0.029\right.$; $\chi^{2}=14.281, p=0.003$ ) (Figure $3 \mathrm{M}-\mathrm{O}$ ).

\section{The Correlation Among M2-Type Macrophages, Tregs and TGF- $\beta /$ Smad Signalling Pathway in CRC}

To explore the relationship among M2-type macrophages, Tregs and TGF- $\beta /$ Smad signalling pathway in CRC, Spearman correlation analysis and linear regression equation were used to compare the expression of CD163, Foxp3 and Smad3. The results showed that the number of M2-type macrophages and Tregs was significantly positively correlated $(\mathrm{r}=0.565, \mathrm{Y}=0.8634 * \mathrm{X}+7.988, p=0.000)$ (Table 3, Figure 4A). Additionally, the number of M2-type macrophages and Tregs was significantly positively correlated with the staining indices of $\operatorname{Smad} 3 \quad(\mathrm{r}=0.657$, $\mathrm{Y}=0.1432 * \mathrm{X}+2.074, \quad p=0.000 ; \quad \mathrm{r}=0.510, \quad \mathrm{Y}=0.08366 * \mathrm{X}$ $+2.425, p=0.000$ ) (Table 4, Figure 4B and C).

\section{The Correlation of the Number of M2-Type Macrophages and Tregs with Tumour Markers in CRC}

Spearman correlation analysis was used to evaluate the correlation of the number of M2-type macrophages and Tregs in CRC with preoperative CEA, CA19-9 and CA724 (Table 5). The number of M2-type macrophages was positively correlated with preoperative CEA, CA19-9 and CA72-4 $(\mathrm{r}=0.466, p=0.000 ; \mathrm{r}=0.218, p=0.001 ; \mathrm{r}=0.178$, $p=0.005)$. The number of Tregs was positively correlated with preoperative CEA and CA19-9 ( $\mathrm{r}=0.386, p=0.000$; $\mathrm{r}=0.144, p=0.023)$, but it had no significant correlation with preoperative CA72-4 $(\mathrm{r}=0.121, p=0.056)$.

\section{Discussion}

TME plays an important role in the development, therapeutic outcomes and clinical regression of malignant tumours. ${ }^{5}$ Both M2-type macrophages and Tregs are important components of TME. It has been found that M2-type macrophages and Tregs are closely related to various malignant tumours, such as ovarian cancer, laryngeal cancer, prostate cancer and nasopharyngeal 


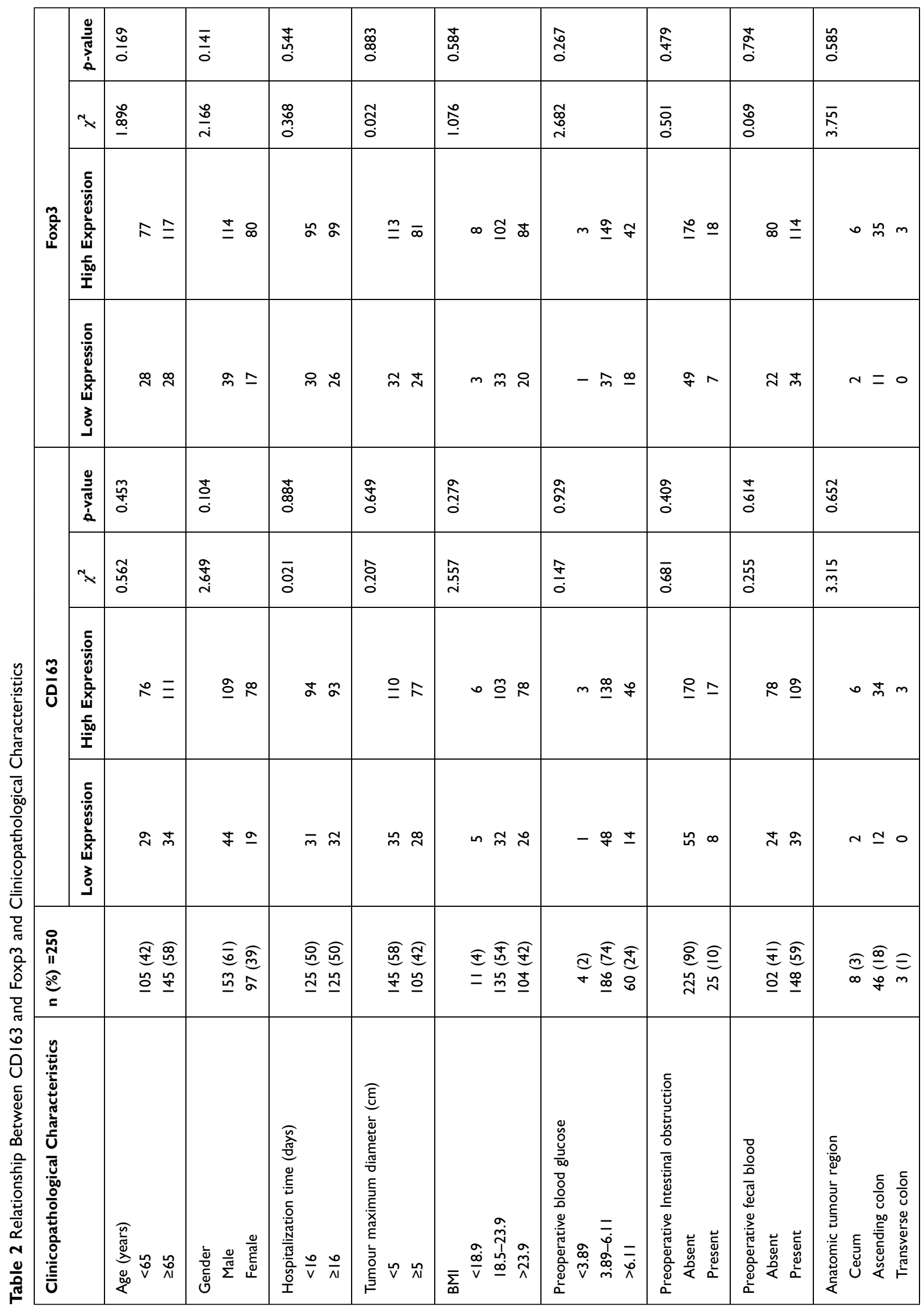




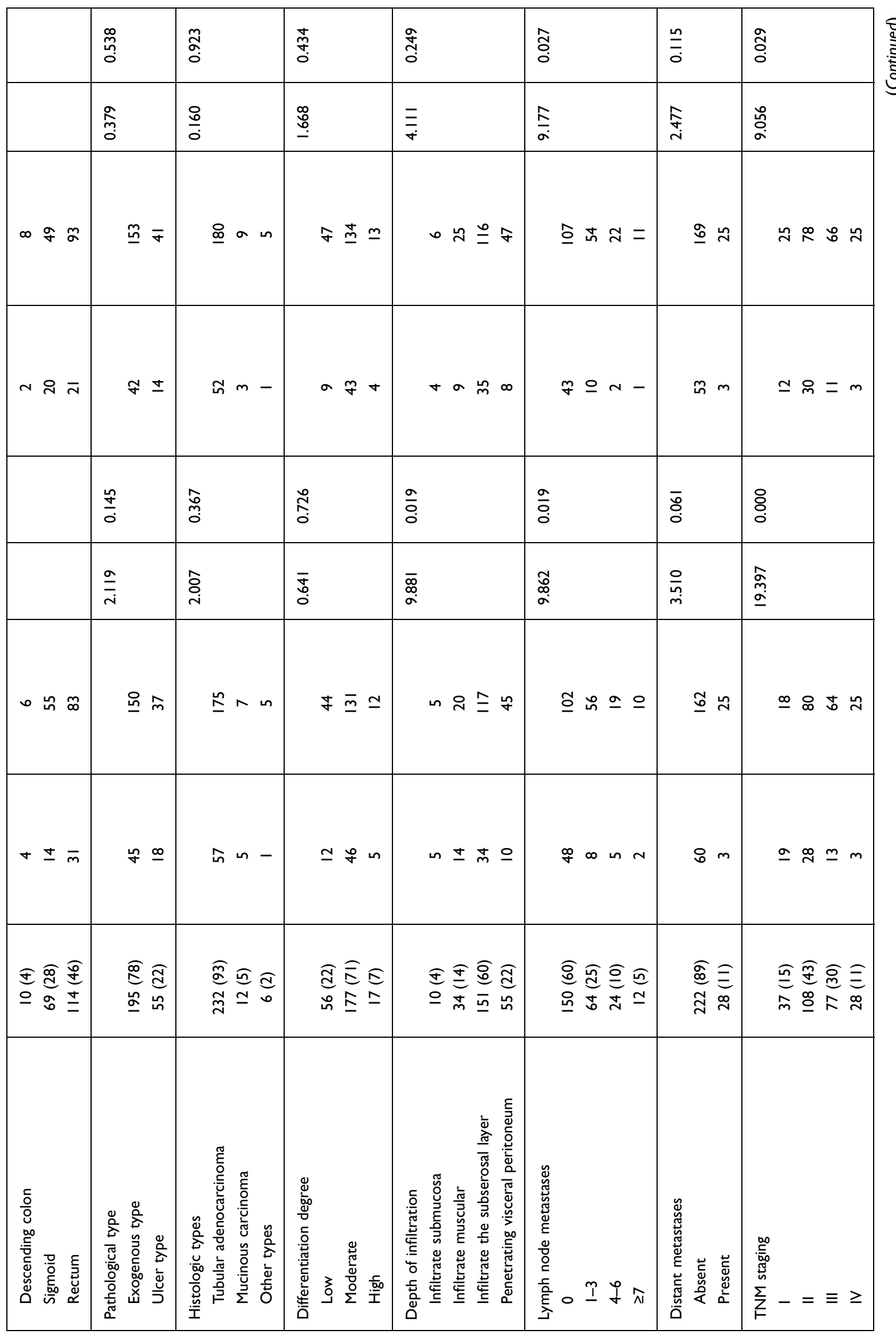




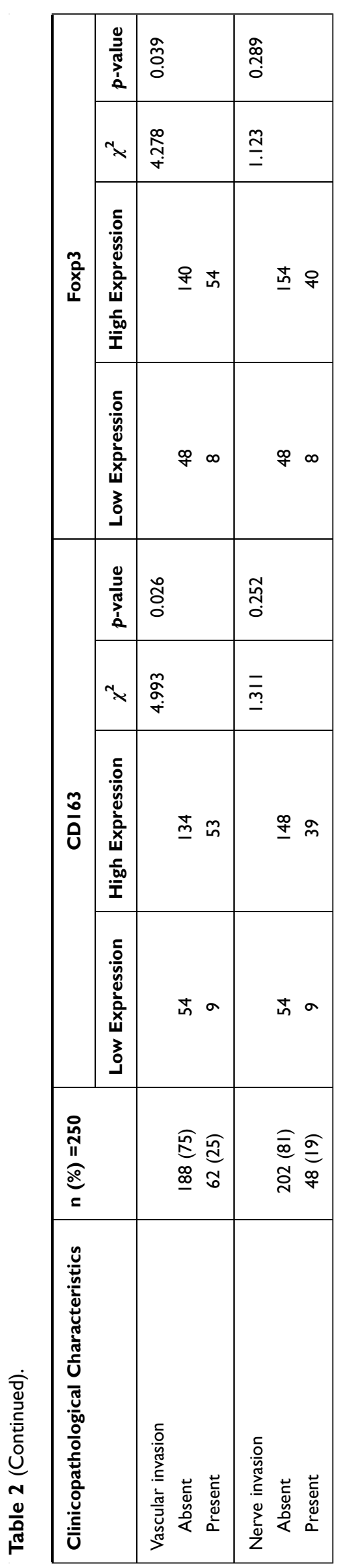

cancer. ${ }^{6,12-14}$ Therefore, exploring the relationship between M2-type macrophages and Tregs has important implications for therapies in CRC. In our study, we found that M2-type macrophages were highly expressed in CRC, and significantly positively correlated with infiltrating Foxp3+ Tregs, which is possibly through activation of the TGF- $\beta$ /Smad signalling pathway, promoting the development of CRC. The above finding has important clinical value for the diagnosis, treatment and prognosis of CRC patients.

Tumour cells can secrete cytokines such as IL-4 and IL-13 to induce the conversion of macrophages to the M2type, which can promote their growth. ${ }^{22,23}$ Thus, tumourassociated macrophages (TAMs) are mainly expressed as M2-type. High infiltration of M2-type macrophages is associated with poor prognosis in almost all malignant tumours. Its main tumour-promoting mechanism is the production of cytokines, such as IL-10 and TGF- $\beta$, which activate tumour cells in relevant signalling pathways, inducing genes associated with cells survival, proliferation and migration, thereby playing an important role in immunosuppression. ${ }^{6,23,24} \mathrm{We}$ found that the expression of M2-type macrophages was upregulated in CRC and significantly correlated with tumour TNM staging, number of lymph node metastases, depth of infiltration and vascular invasion. The above results indicate that M2-type macrophages are involved in the formation of immunosuppressive TME in malignant tumours. Studies ${ }^{19}$ have found that in CRC lung metastases, M2-type macrophages activate the TGF- $\beta /$ Smad signalling pathway by secreting TGF- $\beta$, which is involved in the process of EMT, and the use of TGF- $\beta$ receptor inhibitors can block this signalling pathway to inhibit tumour metastases. Additionally, it has been found that M2-type macrophages promote glioma cell metastases by secreting TGF- $\beta$, activating the Smad2/3 signalling pathway. ${ }^{18}$ We found that the number of M2-type macrophages was significantly positively correlated with the expression of Smad3 in CRC. Thus, it can be hypothesised that M2-type macrophages promote the development of CRC by activating the TGF- $\beta /$ Smad signalling pathway, which is consistent with the previous findings.

In malignant tumours, Tregs inhibit the tumourkilling effects of CD8+ $\mathrm{T}$ and $\mathrm{NK}$ cells through an intercellular contact mechanism or secretion of related cytokines, which in turn affects the immune response of effector $\mathrm{T}$ cells to tumours. ${ }^{9,10,25} \mathrm{We}$ found that the expression of Tregs were upregulated with increasing 

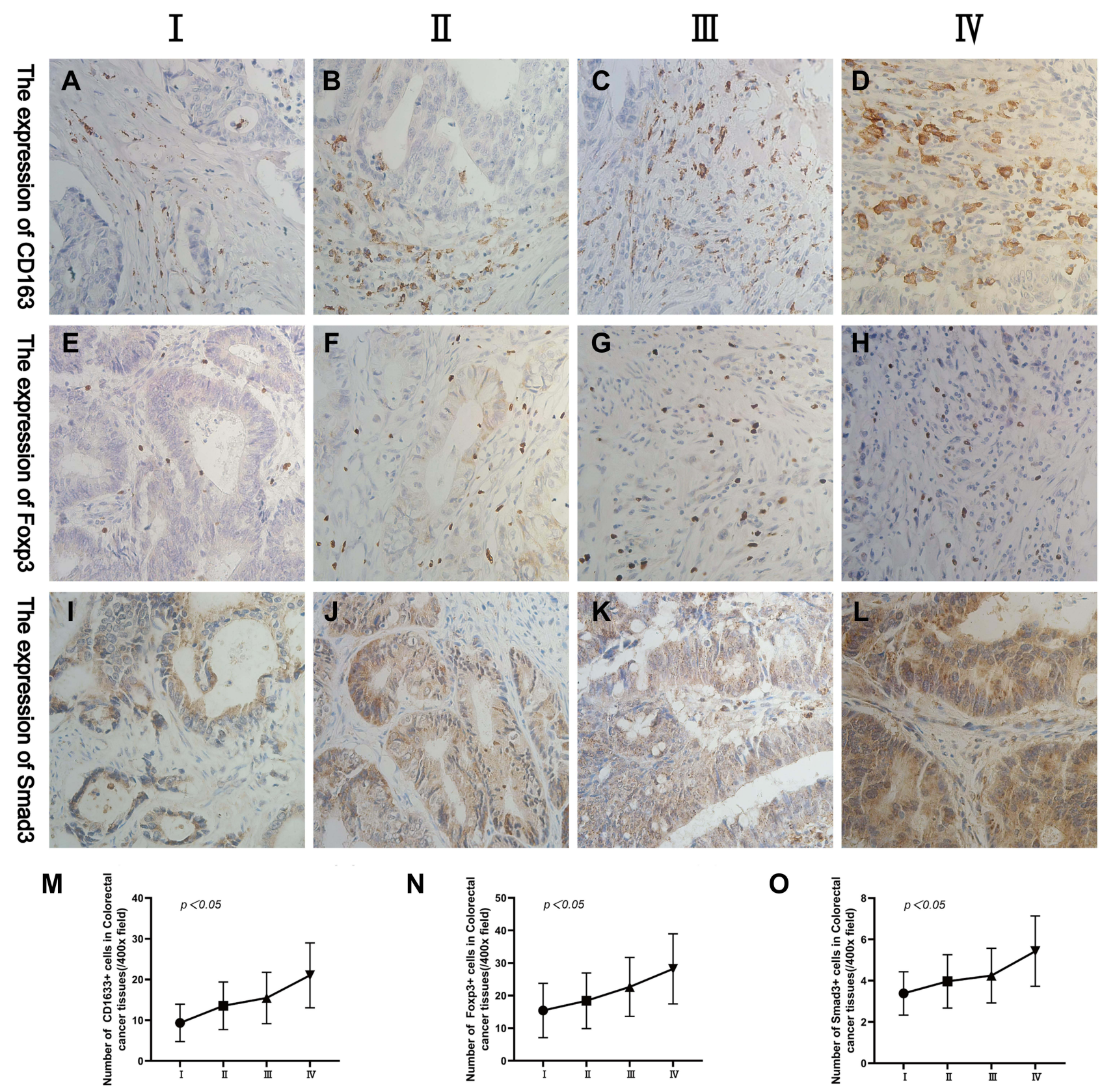

Figure 3 Representative IHC staining of CDI63, Foxp3 and Smad3 in different tumour TNM staging (400x field). (A-D) The expression of CDI63 in CRC at stages I, II, III and IV. (E-H) The expression of Foxp3 in CRC at stages I, II, III and IV. (I-L) The expression levels of Smad3 in CRC at stages I, II, III and IV. (M-O) The changing trend of CDI63, Foxp3 and Smad3 in CRC in different tumour TNM staging.

tumour TNM staging, increasing number of lymph node metastases and increasing vascular invasion, suggesting that Tregs play an important role in the development of

Table 3 Correlation Analysis of CDI63 and Foxp3 Expression in Colorectal Cancer

\begin{tabular}{|l|c|c|}
\hline & & Foxp3 \\
\hline CDI63 & $r$ & 0.565 \\
& $p$ & 0.000 \\
\hline
\end{tabular}

malignant tumours. Tone et $\mathrm{al}^{21}$ found in vitro studies that the relatively conserved enhancer binding site of Foxp3 plays a decisive role in the induction of Foxp3+ Tregs by binding to Smad3 and nuclear factor of activated $\mathrm{T}$ cells (NFAT). Anthoni et $\mathrm{al}^{20}$ used the mouse model with knockout Smad3 gene to study Smad3 in the contact hypersensitivity response and found that Smad3 signalling deficiency can lead to decreased mRNA expression of Foxp3. We found that the expression of Foxp3+ Tregs was upregulated in CRC and significantly 

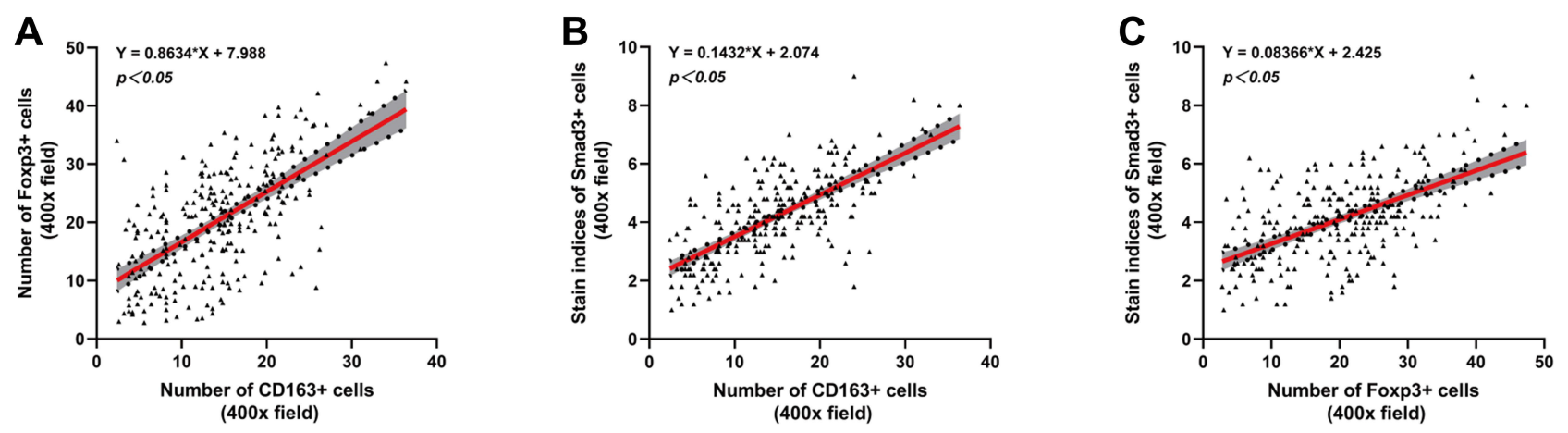

Figure 4 Correlation analysis among CDI63, Foxp3 and Smad3 in CRC. The linear correlation model is shown with a red line. Corresponding $95 \%$ confidence intervals (CI) are shown with the gray area. (A) Correlation between the number of CDI63+ cells and Foxp3+ cells. (B) Correlation between the number of CDI63+ cells and the staining indices of Smad3+ cells. (C) Correlation between the number of Foxp3+ cells and the staining indices of Smad3+ cells.

positively correlated with the expression of Smad3. Combining with previous studies, we can infer that increasing the expression of Smad3 can promote the differentiation of naive $\mathrm{T}$ cells to Foxp $3+$ Tregs. The increase of Tregs can suppress the immune response of effector $\mathrm{T}$ cells to tumours, which in turn promotes CRC development.

The TGF- $\beta /$ Smad signalling pathway is involved in many normal cellular processes, including cell proliferation, differentiation, early development, angiogenesis and wound healing. ${ }^{26,27}$ TGF- $\beta$ has tumour suppressive effects in normal epithelial cells, but affects promoting

Table 4 Correlation Analysis of Smad3 with CDI63 and Foxp3 in Colorectal Cancer

\begin{tabular}{|l|c|c|}
\hline & & Smad3 \\
\hline CDI63 & $r$ & 0.657 \\
& $p$ & 0.000 \\
\hline Foxp3 & $r$ & 0.510 \\
& $p$ & 0.000 \\
\hline
\end{tabular}

Table 5 Correlation Analysis of the Number of M2-Type Macrophages and Tregs with Preoperative CEA, CAI9-9 and CA72-4

\begin{tabular}{|l|c|c|c|c|}
\hline Tumour Markers & \multicolumn{2}{|c|}{$\begin{array}{c}\text { The Mean } \\
\text { Number of } \\
\text { M2-Type } \\
\end{array}$} & $\begin{array}{c}\text { Macrophages } \\
\text { The Mean } \\
\text { Number of } \\
\text { Tregs }\end{array}$ & \multicolumn{2}{|c|}{} \\
\cline { 2 - 5 } & $\mathbf{r}$ & $\boldsymbol{p}$-value & $\mathbf{r}$ & $\boldsymbol{p}$-value \\
\hline Preoperative CEA $(\mu \mathrm{g} / \mathrm{L})$ & 0.466 & 0.000 & 0.386 & 0.000 \\
Preoperative CA19-9 $(\mathrm{U} / \mathrm{mL})$ & 0.218 & 0.001 & 0.144 & 0.023 \\
Preoperative CA72-4 $(\mathrm{U} / \mathrm{mL})$ & 0.178 & 0.005 & 0.121 & 0.056 \\
\hline
\end{tabular}

tumourigenic development in malignant tumours cells. ${ }^{28}$ It is a potent inducer of EMT. ${ }^{16,27}$ Tumour metastases is one of the major causes of death in patients with malignant tumours. EMT is one of the classical modes of tumourigenic invasion and metastases. ${ }^{17,27}$ Many studies ${ }^{29,30}$ have shown that $\operatorname{Smad} 2 / 3$ plays a central role in TGF- $\beta$-induced EMT. At the amino acid level, the most important difference between Smad2 and Smad3 is that the MH1 region of Smad2 has 2 more amino acid fragments than Smad3. Due to the specificity of these 2 amino acid fragments, Smad3 can directly bind to DNA and has transcriptional activity, while Smad2 lacks transcriptional activity. ${ }^{31} \mathrm{Smad} 2 / 3$ has been reported to be mutated in CRC. These mutations have been associated with clinical metastases and low survival rates. $^{32}$ By comparing the expression of Smad3 in normal, paracancerous and cancer tissues, we found that it was significantly increased in CRC. We can speculate that the TGF- $\beta /$ Smad signalling pathway plays an important role in the development of CRC.

Tumour markers have been widely used in CRC research. They play an important role in monitoring recurrence and metastases. ${ }^{33}$ Previous studies ${ }^{4}$ in our laboratory showed that the expression levels of CEA in CRC correlated with the number of M2-type macrophages. Moreover, preoperative CEA, CA19-9 and CA72-4 were also closely associated with CRC lymph node metastases. Combining our above results with previous research, we hypothesised that the expression of M2-type macrophages and Tregs was associated with poor prognosis in CRC.

Previous studies have reported the interaction between M2-type macrophages and Tregs in malignant tumours. We found that the number of M2-type macrophages and Tregs were significantly elevated in CRC tissues compared with normal and paracancerous tissues, indicating that M2-type 
macrophages and Tregs play an important role in tumourigenesis and development. Furthermore, the expression levels of M2-type macrophages and Tregs was significantly positively correlated in CRC. We can speculate that the interaction between M2-type macrophages and Tregs can promote the occurrence and development of $\mathrm{CRC}$, which is consistent with previous studies in other malignant tumours. Sun et $\mathrm{al}^{34}$ found that in the co-culture of naive $\mathrm{T}$ cells with M2-type macrophages in lung disease, M2-type macrophages could promote naive $\mathrm{T}$ cells differentiation toward Foxp3+ Tregs. Furthermore, the addition of the TGF- $\beta$ / Smad signalling pathway inhibitor LY2109761 resulted in a reduced proportion of Tregs comparing to the previous ones. These results suggest that the TGF- $\beta /$ Smad signalling pathway is involved in the differentiation process of Tregs induced by M2-type macrophages. Our study found that the expression of Smad3 was significantly elevated in $\mathrm{CRC}$, suggesting that the TGF- $\beta$ /Smad signalling pathway is aberrantly activated during tumourigenesis and development, promoting EMT and tumour metastases. Smad3 was significantly positively correlated with the expression levels of M2-type macrophages and Tregs, respectively, suggesting that M2-type macrophages play a synergistic role with Tregs in CRC, and the TGF- $\beta /$ Smad signalling pathway may play a pivotal role. The exact relationship between M2type macrophages and Tregs needs further study. In the future, we plan to further investigate the correlation between M2 macrophages and Tregs by blocking the TGF- $\beta /$ Smad signalling pathway and observe the difference in expression between M2-type macrophages and Tregs after blocking, which can clarify their potential role as immunotherapeutic targets for CRC.

\section{Conclusions}

M2-type macrophages may activate the TGF- $\beta /$ Smad signalling pathway by secreting TGF- $\beta$, which leads to increased expression of Foxp3+ Tregs and further promotes the tumourigenesis and development of CRC. M2type macrophages, Tregs and TGF- $\beta /$ Smad signalling pathway may become potential targets for novel immunotherapy of $\mathrm{CRC}$, which has important clinical value for the diagnosis, treatment and prognosis of CRC patients.

\section{Abbreviations}

Tregs, regulatory T cells; CRC, colorectal cancer; CD163, cluster of differentiation 163; Foxp3, forkhead transcription factor $\mathrm{p} 3$; Smad3, drosophila mothers against decapentaplegic protein 3; TME, tumour microenvironment; EMT, epithelial-mesenchymal-transition; TGF- $\beta$, transforming growth factor- $\beta$; IHC, immunohistochemical; DAB, 3,3'-diaminobenzidine; PBS, phosphate buffered saline; mRNA, messenger ribonucleic acid.

\section{Data Sharing Statement}

The data that support the findings of this study are available on request from the corresponding author (Dr. Qiao).

\section{Ethics Approval and Informed Consent}

The study was conducted in accordance with the Ethics Committee of the First Affiliated Hospital of Jinzhou Medical University (KYLL 202089) and the 1964 Helsinki Declaration. Informed consent was obtained from all participants included in the study.

\section{Acknowledgments}

The authors sincerely appreciate the assistance of The First Affiliated Hospital of Jinzhou Medical University.

\section{Author Contributions}

All authors made a significant contribution to the work reported, whether that is in the conception, study design, execution, acquisition of data, analysis and interpretation, or in all these areas; took part in drafting, revising or critically reviewing the article; gave final approval of the version to be published; have agreed on the journal to which the article has been submitted; and agree to be accountable for all aspects of the work.

\section{Funding}

There is no funding to report.

\section{Disclosure}

The authors have declared that no competing interest exists.

\section{References}

1. Sawicki T, Ruszkowska M, Danielewicz A, et al. A review of colorectal cancer in terms of epidemiology, risk factors, development, symptoms and diagnosis. Cancers (Basel). 2021;13:2025. doi: $10.3390 /$ cancers 13092025

2. Elia I, Haigis MC. Metabolites and the tumour microenvironment: from cellular mechanisms to systemic metabolism. Nat Metab. 2021;3:21-32. doi:10.1038/s42255-020-00317-z 
3. Martinez-Reyes I, Chandel NS. Cancer metabolism: looking forward. Nat Rev Cancer. 2021;21(10):669-680. doi:10.1038/s41568-02100378-6

4. Wang Y, Wang J, Yang C, et al. A study of the correlation between M2 macrophages and lymph node metastasis of colorectal carcinoma. World J Surg Oncol. 2021;19:91. doi:10.1186/s12957-021-02195-5

5. Quail DF, Joyce JA. Microenvironmental regulation of tumor progression and metastasis. Nat Med. 2013;19:1423-1437. doi:10.1038/ nm.3394

6. Wang J, Huang H, Lu J, et al. Tumor cells induced-M2 macrophage favors accumulation of Treg in nasopharyngeal carcinoma. Int J Clin Exp Pathol. 2017;10:8389-8401.

7. Wu Y, Yang S, Ma J, et al. Spatiotemporal immune landscape of colorectal cancer liver metastasis at single-cell level. Cancer Discov. 2021:candisc.0316.2021. doi:10.1158/2159-8290.CD-21-0316

8. Shrivastava R, Shukla N. Attributes of alternatively activated (M2) macrophages. Life Sci. 2019;224:222-231. doi:10.1016/j.lfs.2019. 03.062

9. Cinier J, Hubert M, Besson L, et al. Recruitment and expansion of Tregs cells in the tumor environment-how to target them? Cancers (Basel). 2021;13(8):1850. doi:10.3390/cancers 13081850

10. Vignali DA, Collison LW, Workman CJ. How regulatory T cells work. Nat Rev Immunol. 2008;8:523-532. doi:10.1038/nri2343

11. Fontenot JD, Gavin MA, Rudensky AY. Foxp3 programs the development and function of $\mathrm{CD} 4+\mathrm{CD} 25+$ regulatory $\mathrm{T}$ cells. Nat Immunol. 2003;4:330-336. doi:10.1038/ni904

12. Zhu $\mathrm{Q}, \mathrm{Wu} \mathrm{X}, \mathrm{Wu} \mathrm{Y}$, et al. Interaction between Treg cells and tumor-associated macrophages in the tumor microenvironment of epithelial ovarian cancer. Oncol Rep. 2016;36:3472-3478. doi:10.3892/or.2016.5136

13. Sun W, Wei FQ, Li WJ, et al. A positive-feedback loop between tumour infiltrating activated Treg cells and type 2-skewed macrophages is essential for progression of laryngeal squamous cell carcinoma. $B r \quad J$ Cancer. 2017;117:1631-1643. doi:10.1038/ bjc. 2017.329

14. Erlandsson A, Carlsson J, Lundholm M, et al. M2 macrophages and regulatory T cells in lethal prostate cancer. Prostate. 2019;79: 363-369. doi: $10.1002 /$ pros. 23742

15. Zhang N, Ng AS, Cai S, et al. Novel therapeutic strategies: targeting epithelial-mesenchymal transition in colorectal cancer. Lancet Oncol. 2021;22:e358-e368. doi:10.1016/S1470-2045(21)00343-0

16. Derynck R, Muthusamy BP, Saeteurn KY. Signaling pathway cooperation in TGF-beta-induced epithelial-mesenchymal transition. Curr Opin Cell Biol. 2014;31:56-66. doi:10.1016/j.ceb.2014.09.001

17. Wang X, Lai Q, He J, et al. LncRNA SNHG6 promotes proliferation, invasion and migration in colorectal cancer cells by activating TGF-beta/Smad signaling pathway via targeting UPF1 and inducing EMT via regulation of ZEB1. Int J Med Sci. 2019;16:51-59. doi:10.7150/ijms. 27359

18. Liu Z, Kuang W, Zhou Q, et al. TGF-beta1 secreted by M2 phenotype macrophages enhances the stemness and migration of glioma cells via the SMAD2/3 signalling pathway. Int $J$ Mol Med. 2018;42:3395-3403. doi:10.3892/ijmm.2018.3923
19. Cai J, Xia L, Li J, et al. Tumor-associated macrophages derived TGF-betaInduced epithelial to mesenchymal transition in colorectal cancer cells through Smad2,3-4/Snail signaling pathway. Cancer Res Treat. 2019;51:252-266. doi:10.4143/crt.2017.613

20. Anthoni M, Fyhrquist-Vanni N, Wolff H, et al. Transforming growth factor-beta/Smad3 signalling regulates inflammatory responses in a murine model of contact hypersensitivity. $\mathrm{Br} J$ Dermatol. 2008;159:546-554. doi:10.1111/j.1365-2133.2008.08696.x

21. Tone Y, Furuuchi K, Kojima Y, et al. Smad3 and NFAT cooperate to induce Foxp3 expression through its enhancer. Nat Immunol. 2008;9:194-202. doi:10.1038/ni1549

22. Murray PJ, Wynn TA. Protective and pathogenic functions of macrophage subsets. Nat Rev Immunol. 2011;11:723-737. doi:10.1038/ nri3073

23. Shapouri-Moghaddam A, Mohammadian S, Vazini H, et al. Macrophage plasticity, polarization, and function in health and disease. J Cell Physiol. 2018;233:6425-6440. doi:10.1002/jcp.26429

24. Xue T, Yan K, Cai Y, et al. Prognostic significance of CD163+ tumor-associated macrophages in colorectal cancer. World J Surg Oncol. 2021;19:186. doi:10.1186/s12957-021-02299-y

25. Sundstrom P, Stenstad H, Langenes V, et al. Regulatory T cells from colon cancer patients inhibit effector T-cell migration through an adenosine-dependent mechanism. Cancer Immunol Res. 2016;4:183-193. doi:10.1158/2326-6066.CIR-15-0050

26. David CJ, Massague J. Contextual determinants of TGFbeta action in development, immunity and cancer. Nat Rev Mol Cell Biol. 2018;19:419-435. doi:10.1038/s41580-018-0007-0

27. Hao Y, Baker D, Ten Dijke P. TGF-beta-mediated epithelial-mesenchymal transition and cancer metastasis. Int $J$ Mol Sci. 2019;20(11):2767. doi:10.3390/ijms20112767

28. Gupta S, Maitra A. EMT: matter of life or death? Cell. 2016;164:840-842. doi:10.1016/j.cell.2016.02.024

29. Massague J. TGFbeta in Cancer. Cell. 2008;134:215-230. doi:10.1016/j.cell.2008.07.001

30. Chen HY, Chiang YF, Huang JS, et al. Isoliquiritigenin reverses epithelial-mesenchymal transition through modulation of the TGF-beta/Smad signaling pathway in endometrial cancer. Cancers (Basel). 2021;13. doi:10.3390/cancers13061236

31. Shi Y, Wang YF, Jayaraman L, et al. Crystal structure of a Smad MH1 domain bound to DNA: insights on DNA binding in TGF-beta signaling. Cell. 1998;94:585-594. doi:10.1016/s0092-8674(00)81600-1

32. Chen Y, Xing P, Chen Y, et al. High p-Smad2 expression in stromal fibroblasts predicts poor survival in patients with clinical stage I to IIIA non-small cell lung cancer. World J Surg Oncol. 2014;12:328. doi:10.1186/1477-7819-12-328

33. Li Q, Dai W, Li Y, et al. Nomograms for predicting the prognostic value of serological tumor biomarkers in colorectal cancer patients after radical resection. Sci Rep. 2017;7:46345. doi:10.1038/srep46345

34. Sun SW, Chen L, Zhou M, et al. BAMBI regulates macrophages inducing the differentiation of Treg through the TGF-beta pathway in chronic obstructive pulmonary disease. Respir Res. 2019;20:26. doi:10.1186/s12931-019-0988-z
OncoTargets and Therapy

\section{Publish your work in this journal}

OncoTargets and Therapy is an international, peer-reviewed, open access journal focusing on the pathological basis of all cancers, potential targets for therapy and treatment protocols employed to improve the management of cancer patients. The journal also focuses on the impact of management programs and new therapeutic agents and protocols on patient perspectives such as quality of life, adherence and satisfaction. The manuscript management system is completely online and includes a very quick and fair peer-review system, which is all easy to use. Visit http://www.dovepress.com/ testimonials.php to read real quotes from published authors. 